\title{
Clinical efficacy of bipolar radiofrequency ablation of small renal masses
}

\author{
S. G. C. Kroeze • M. Agenant • G. N. Jonges $\cdot$ T. Stein $\cdot$ \\ J. L. H. R. Bosch
}

Received: 28 July 2014 / Accepted: 16 October 2014 / Published online: 29 October 2014

(C) Springer-Verlag Berlin Heidelberg 2014

\begin{abstract}
Purpose The new development of bipolar radiofrequency ablation (RFA) can overcome problems observed with monopolar RFA for the treatment of small renal masses (SRM). Energy is more homogeneously delivered, and higher current densities can be used. Data on treatment of renal tumors with bipolar RFA are still limited. The aim of this study was to examine the clinical efficacy of bipolar multiprobe RFA for treatment of SRM, according to the IDEAL recommendations.

Methods Ten SRMs in 10 consecutive patients were ablated using multipolar RFA. Outcome measures were technical success, applied energy, and observed complications. Hereafter, tumors were excised in an open surgical fashion and histologically analyzed for RFA lesion volume and presence of viable cells.

Results Median patient age was 59.5 (range 39.2-69.8) years. Median tumor diameter was 2.5 (range 1.6-4.5) $\mathrm{cm}$. Technical success rate was $100 \%$. In five procedures, two probes were used, and in five procedures three probes were used. Median ablation time was 18 (range 12-38) minutes in which a median of 30.5 (range 23.6-102) kJ was applied. Complications included one patient who developed a urinoma. Median ablated volume was $4.4(2.2-29.9) \mathrm{cm}^{3}$. In
\end{abstract}

S. G. C. Kroeze $(\varangle) \cdot$ M. Agenant · J. L. H. R. Bosch Department of Urology, University Medical Center Utrecht, Heidelberglaan 100, 3584 CX Utrecht, The Netherlands e-mail: s.kroeze@jbz.nl

G. N. Jonges

Department of Pathology, University Medical Center Utrecht, Utrecht, The Netherlands

T. Stein

Olympus Surgical Technologies Europe, Teltow, Germany all cases, the ablated volume was larger than the tumor. No viable cells were present within the ablated tissue.

Conclusions Multipolar RFA is clinically successful for treating SRMs. Using preoperatively calculated energy settings, tailored size tumor lesions could be created. Clinical efficacy and oncological outcomes need to be investigated further in studies using multipolar RFA in a percutaneous fashion.

Keywords Radiofrequency ablation · Bipolar . Multiprobe $\cdot$ Small renal masses $\cdot$ Kidney cancer

\section{Abbreviations \\ RFA Radiofrequency ablation \\ SRM Small renal masses}

\section{Introduction}

Radiofrequency ablation (RFA) for small renal masses (SRM) has become a commonly used nephron-sparing treatment in selected elderly and comorbid patients [1, 2]. So far, monopolar RFA [3] has been the principal approach for RFA. One of the most important downsides of monopolar RFA is that thermal energy can be lost through the "heat sink' effect and 'skipping' [4-6]. Also, when using a single electrode, the lesion size remains limited [7]. These factors result in significant differences in size and shape of ablated lesions, despite identical energy delivery with monopolar RFA [8-10]. Therefore, it may be difficult to accurately target a renal lesion.

This study examined the use of bipolar multipolar RFA as treatment for SRM. One major advantage of bipolar RFA is that it is an isolated electrical circuit [11]. Current travels through the tissue between the electrodes and 
not through the whole body to the return plate as with monopolar RFA devices. That means that energy deposition can be better controlled, is more homogeneously applied, and higher current densities can be used. Furthermore, the probes are continuously cooled by internal perfusion, which reduces tissue resistance, and this system allows the use of up to three probes with six electrodes at the same time that can interact with each other. These factors combined are intended to result in larger lesions with a more uniform shape [12] and a reliable dose-effect relationship [13, 14].

Advanced clinical experience with bipolar multiprobe RFA has primarily been obtained in the treatment of liver metastases [15-17], while its use for treatment of renal masses is currently described in only a few clinical studies [18-22]. To objectively assess the value of bipolar multiprobe RFA, the IDEAL recommendations were used [23]. Bipolar RFA has completed the first stage of the IDEAL model (innovation) and has now arrived at stage 2a (development). This stage focuses on the description of the procedure, technical modifications, and clear outcome reporting in a small set of patients and is intended to increase experience and investigate indications of use.

The aim of this study was to prospectively investigate the energy settings of a multiprobe bipolar RFA device that are needed to successfully ablate SRM, according to the IDEAL recommendations. The primary objective is technical success in the form of achieved ablation area size, clinical efficacy, and observed complications.

\section{Materials and methods}

This study has been approved by the local research ethics committee of the University Medical Center Utrecht and has therefore been performed in accordance with the ethical standards laid down in the 1964 Declaration of Helsinki. All patients gave their informed consent prior to their inclusion in the study. Ten consecutive patient with an indication for open partial nephrectomy for a small renal mass (SRM) participated in the study at the University Medical Center Utrecht. Prior to surgery, SRM were confirmed to be renal cell carcinoma (RCC) by renal biopsy. Inclusion criteria were patients with small cortical RCCs $(\leq 4 \mathrm{~cm})$ on CT imaging. Exclusion criteria were patients $<18$ years old, pregnant women, renal tumors positioned in the kidney hilus, benign tumors, coagulation disorders or use of anticoagulants that could not be temporarily stopped.

With use of an abdominal CT scan with intravenous contrast, the maximum diameters $(\mathrm{a}=$ length, $\mathrm{b}=$ width, and $\mathrm{c}=$ heigth) of the tumor were manually measured. The required safety margin (s) of $5 \mathrm{~mm}$ was added to the diameters. The target volume $\mathrm{V}\left(\mathrm{cm}^{3}\right)$ necessary for complete ablation was calculated using the equation: $\mathrm{V}=(\pi / 6) \times(\mathrm{a}+\mathrm{s}) \times(\mathrm{b}+\mathrm{s}) \times(\mathrm{c}+\mathrm{s})$. The target energy $E(k J)$ to be applied was calculated using the equation $\mathrm{E}=2 \times \mathrm{V}$ (where $\mathrm{V}$ is the volume in $\mathrm{ml}$, and $\mathrm{E}$ is the energy in $\mathrm{kJ})$. The factor 2 is based on experience with the multipolar ablation of liver metastases [16]. The number and type of the electrodes depends on the target coagulation diameter; for a diameter smaller than $2 \mathrm{~cm}$ one probe was used, a diameter between 2 and $3 \mathrm{~cm}$ required two probes, and a diameter more than 3 required three probes. Probes were placed centrally in the tumor. The distance between the probes was based on data from a previous study by Frericks et al. [16]. For $230-\mathrm{W}$ probes, the distance was $13 \mathrm{~mm}$, for $330-\mathrm{W}$ probes, the distance was $15 \mathrm{~mm}$, and for $340-\mathrm{W}$ probes the distance was $20 \mathrm{~mm}$.

All patients underwent operative exploration and open partial nephrectomy through a transperitoneal subcostal incision, all operations were performed by one urologist (JLHRB). After kidney mobilization, two to three probes were inserted at the calculated locations within the tumor perpendicular to the tumor surface (Fig. 1). The kidney was covered with wet sterile gauzes to prevent dehydration. Probes with active conductors of either 30 or $40 \mathrm{~mm}$ (Celon ProSurge T30 or T40, Olympus Surgical Technologies Europe, Germany) were used. For the 30-mm active conductors, a power level of $30 \mathrm{~W}$ per probe was selected (two probes: $60 \mathrm{~W}$; three probes: $90 \mathrm{~W}$ ), for the $40 \mathrm{~mm}$ active conductors $40 \mathrm{~W}$ per probe (three probes: $120 \mathrm{~W}$ ). RFA was performed using a power-control system (CelonLab Power, Olympus Surgical Technologies Europe, Germany). This system produces a maximum power of $250 \mathrm{~W}(470 \mathrm{kHz})$ and is feedback controlled by measuring tissue resistance. During the procedure, rise of resistance was prevented by internally cooling the probes with continuously flowing $\mathrm{NaCl} 0.9 \%$ at room temperature. Every probe has two electrodes and is bipolar; up to three probes can be connected to the power-control system. When two or three probes are inserted at the same time, it becomes a multipolar system [11]. The probes were removed, and the ablated tumor was excised. Partial nephrectomy was performed according to standard medical practice, and the tissue specimen was sent to the pathologist for further evaluation.

The resected tissue was cut transversely into slices of $5 \mathrm{~mm}$ perpendicular to the needle track. Slices were formalin-fixated and paraffin-embedded (FFPE) or snapfrozen in turns. FFPE material with a slice thickness of $10 \mu \mathrm{m}$ was stained with haematoxylin and eosin, and frozen tissue was nicotinamide adenine dinucleotide (NADH) stained in order to analyze cellular viability within the 

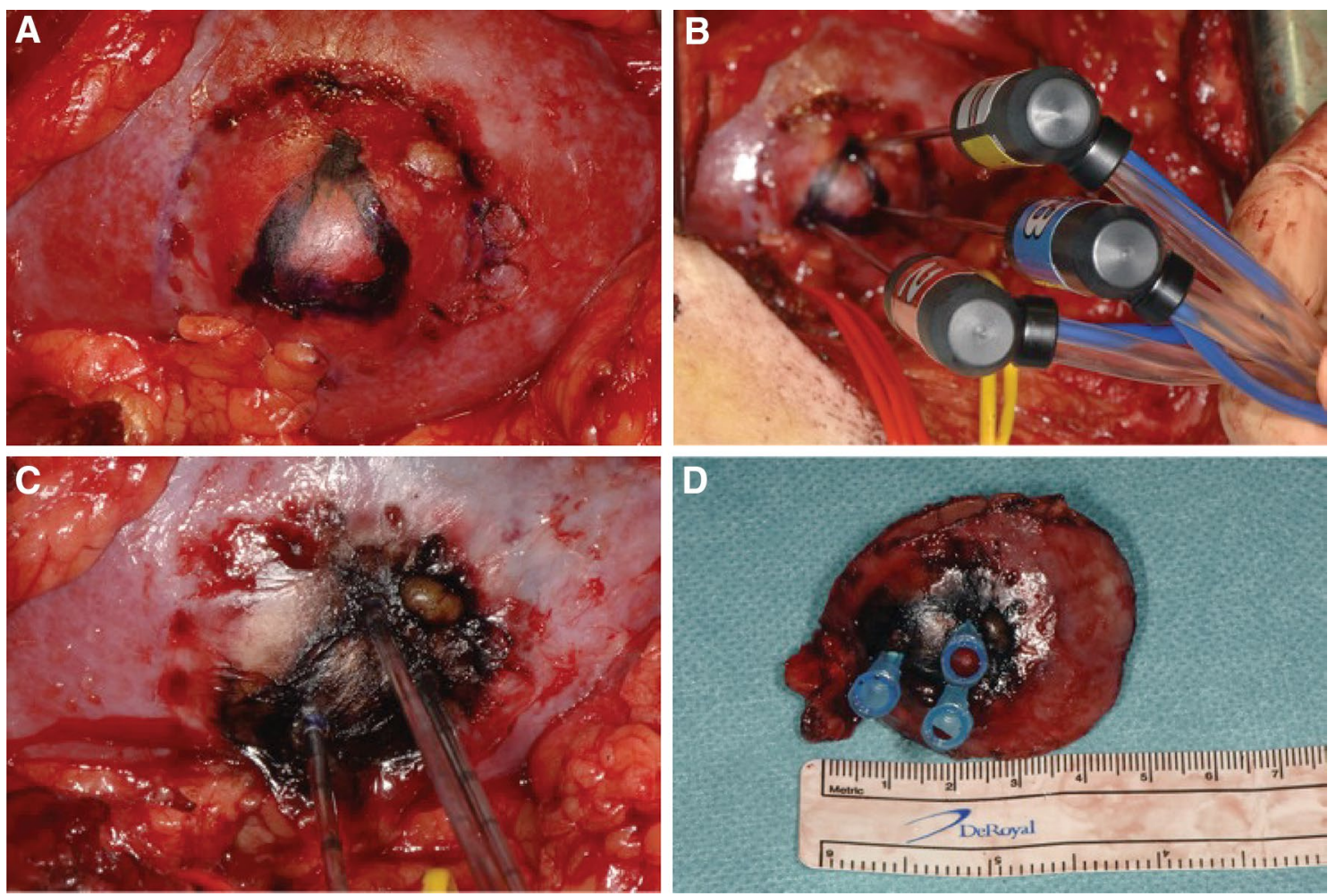

Fig. 1 a The SRM is surgically exposed, b the RFA probes are placed according to preoperatively calculated distances, $\mathbf{c}$ the tumor is ablated using multipolar RFA, $\mathbf{d}$ the tumor is surgically excised

Table 1 Patient and tumor characteristics and results of RFA procedures

\begin{tabular}{|c|c|c|c|c|c|c|c|c|c|c|c|}
\hline $\begin{array}{l}\text { Patient } \\
\text { Sex }\end{array}$ & $\begin{array}{l}1 \\
\text { Female }\end{array}$ & $\begin{array}{l}2 \\
\text { Male }\end{array}$ & $\begin{array}{l}3 \\
\text { Male }\end{array}$ & $\begin{array}{l}4 \\
\text { Male }\end{array}$ & $\begin{array}{l}5 \\
\text { Male }\end{array}$ & $\begin{array}{l}6 \\
\text { Male }\end{array}$ & $\begin{array}{l}7 \\
\text { Male }\end{array}$ & $\begin{array}{l}8 \\
\text { Female }\end{array}$ & $\begin{array}{l}9 \\
\text { Male }\end{array}$ & $\begin{array}{l}10 \\
\text { Male }\end{array}$ & Median (range) \\
\hline Age (years) & 42.2 & 63.2 & 68.7 & 67.9 & 66.0 & 55.9 & 69.8 & 39.2 & 49.4 & 50.1 & $59.5(39.2-69.8)$ \\
\hline Tumor diameter $(\mathrm{cm})$ & 3.1 & 1.6 & 4.5 & 2.5 & 2.6 & 1.8 & 1.9 & 1.8 & 2.8 & 2.4 & $2.5(1.6-4.5)$ \\
\hline Side of tumor & Left & Left & Left & Left & Right & Left & Right & Left & Right & Left & \\
\hline PADUA score & 7 & 8 & 13 & 11 & 7 & 8 & 8 & 9 & 6 & 6 & \\
\hline Number of probes & 2 & 2 & 3 & 3 & 3 & 2 & 2 & 2 & 3 & 3 & \\
\hline Applied energy (kJ) & 25.8 & 23.6 & 102 & 46.2 & 51.0 & 30.1 & 30.8 & 29.3 & 57.9 & 27.8 & $30.5(23.6-102)$ \\
\hline Ablated volume $\left(\mathrm{cm}^{3}\right)$ & 6.8 & 4.4 & 29.9 & 9.4 & 9.7 & 4.3 & 4.2 & 2.9 & 4.0 & 2.2 & $4.4(2.2-29.9)$ \\
\hline Tumor volume $\left(\mathrm{cm}^{3}\right)$ & 3.8 & 1.3 & 13.4 & 5.2 & 4.3 & 1.4 & 1.8 & 1.5 & 4.0 & 2.0 & $2.9(1.3-13.4)$ \\
\hline Effective safety margin $(\mathrm{cm})$ & 0.3 & 0.4 & 0.4 & 0.2 & 0.3 & 0.3 & 0.3 & 0.2 & 0.1 & 0.1 & $0.3(0.1-0.4)$ \\
\hline Complications & 0 & 0 & $1^{\mathrm{a}}$ & 0 & 0 & 0 & 0 & 0 & 0 & 0 & \\
\hline Histology & $\mathrm{ccRCC}$ & $\mathrm{ccRCC}$ & $\mathrm{ccRCC}$ & $\mathrm{ccRCC}$ & $\mathrm{ccRCC}$ & $\mathrm{ccRCC}$ & $\mathrm{ccRCC}$ & $\mathrm{ccRCC}$ & $\mathrm{pRCC}$ & $\mathrm{ccRCC}$ & \\
\hline
\end{tabular}

a Urinoma

ablated area [6]. The totally ablated area and the ablated tumor size were measured using the maximum diameter of the needle axis (VL), the long axis (DL), and short axis (SL) in the transverse plane to the needle. The ablated volumes were calculated using the formula $\mathrm{V}=(\pi / 6) \times(\mathrm{VL}$ $\times \mathrm{DL} \times \mathrm{SL})$.

\section{Results}

Patient and tumor characteristics as well as results of the RFA procedures are shown in Table 1. Ten renal masses were treated in 10 patients. Median age of the study population was 59.5 (range 39.2-69.8) years. Median tumor 

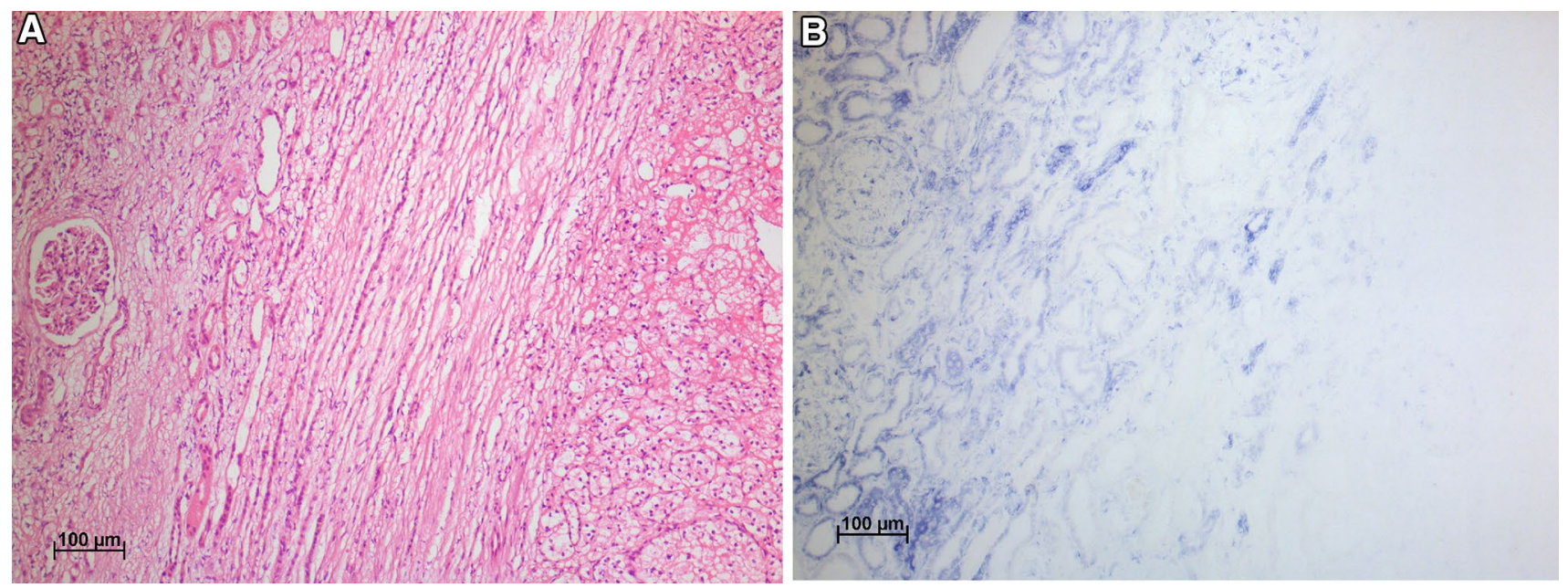

Fig. 2 Histology of an ablated tumor. a H\&E staining of the edge of an ablated tumor. b NADH staining of the equivalent slide to the H\&E staining. White indicates nonviable tissue, blue indicates viable tissue
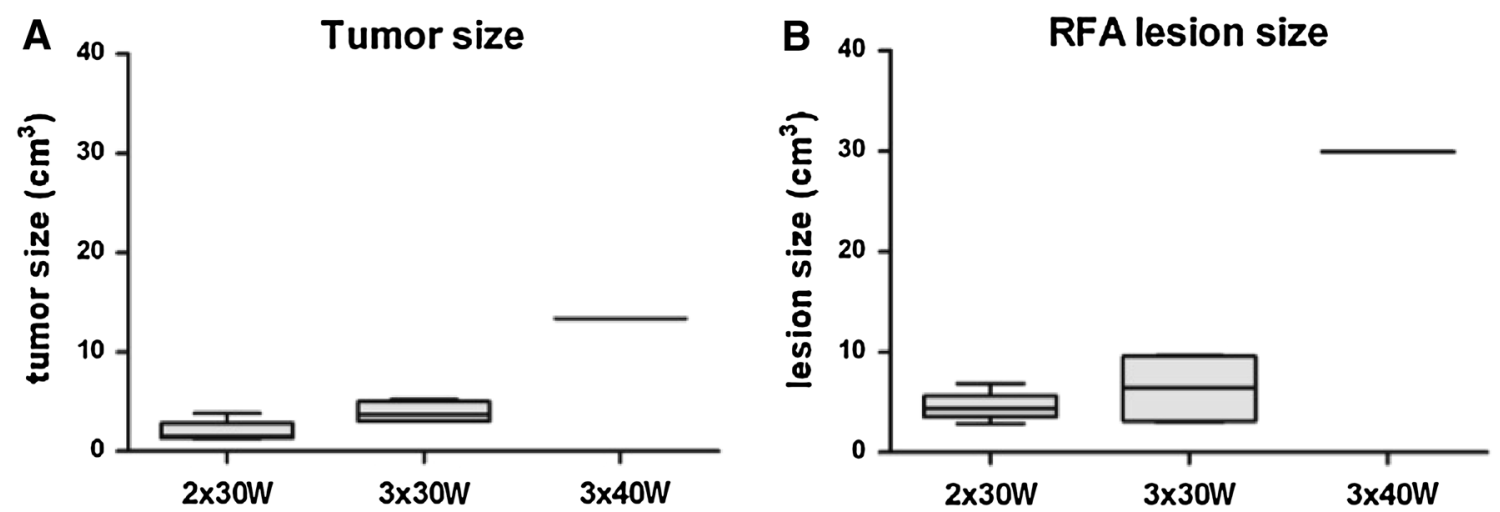

Fig. 3 a Tumor volumes subdivided into preoperatively calculated energy settings. b RFA lesion volume following RFA using the preoperatively calculated energy settings. $2 \times 30 \mathrm{~W}: n=5,3 \times 30 \mathrm{~W}: n=4,3 \times 40 \mathrm{~W}: n=1$

diameter was 2.5 (range 1.6-4.5) $\mathrm{cm}$. All tumors were clinically scored as T1aNOM0 (TNM 2002 classification) before inclusion. The anatomical characteristics were scored using the PADUA classification [24]. Multipolar RFA was performed in all procedures. In five procedures, two probes were used, and in five procedures, three probes were used. All ablation procedures were performed in one cycle. The median duration of ablation was 18 (range 12 38 ) minutes, in which a median of 30.5 (range 23.6-102) kJ was applied. Technical success rate was $100 \%$. There were no intraoperative complications (e.g., damage to surrounding organs) due to the RFA procedure. There was one postoperative complication consisting of a persistent urinoma due to a persistent urinary fistula, for which this patient eventually underwent a nephrectomy. After staining with $\mathrm{NADH}$, no viable cells were observed within the ablated area of all treated tumors (Fig. 2). The median histologically measured ablated volume was 4.4 (2.2-29.9 range) $\mathrm{cm}^{3}$. The median histologically measured tumor volume was 2.9 (1.3-13.4 range) $\mathrm{cm}^{3}$. Subgroup analysis of multiprobe RFA with 2 probes versus multiprobe RFA with 3 probes is shown in Fig. 3. This shows that the lesion size is always larger than the tumor size and that for every tumor size a tailored preoperative energy setting can be created.

\section{Discussion}

We studied the use of multiprobe bipolar RFA for SRM using the IDEAL recommendations (stage 2a; development). In this study, the preoperatively determined energy settings were reliable, resulting in a larger ablated volume than the size of the tumor in all procedures. The median diameter of the ablated tissue mass was $0.6 \mathrm{~cm}$ (range $0.2-0.8$ ) larger than that of the tumor mass, which indicates that there is a safety margin of $0.3 \mathrm{~cm}$ surrounding 
the tumor. Furthermore, there was no presence of viable tumor cells within the ablated area. Although studied in small patient populations, with a variety of types of probes and a relatively short follow-up (IDEAL stage 1; innovation and stage $2 \mathrm{a}$; development), the few studies that performed bipolar or multipolar RFA on renal tumors indicate that similar lesion volumes were created in a percutaneous or laparoscopic fashion [18-22], and no recurrence was observed (follow-up range 0-36 months), or no viable cells were found within the ablated area. In these studies, ablation volumes of 7.1-86.5 $\mathrm{cm}^{3}$ were obtained, depending on the size of the treated tumor. It has previously been shown that simultaneous application of multiple probes is significantly more effective than sequentially applied probes with a similar amount of applied energy [25]. Our study shows that by using this technique, tumors could be treated with a preoperatively determined tailored energy dose (Fig. 3). With the increase in the number of probes to 4,5 , or 6 , which is possible using special adapters, theoretically much larger lesion volumes could be created [26]. However, this needs to be further examined in clinical trials.

Furthermore, it needs to be examined whether the location of the tumor influences the chance of complications, as present studies show contradictory results [27, 28]. One patient in our series developed a urinoma because of a persistent urinary fistula. A urinary fistula is a well-known complication of partial nephrectomy and usually closes spontaneously or after Double-J stent drainage. This tumor had a PADUA score of 13 and was located closely to the urinary collecting system. Although it is known that this tumor is a complex case for standard partial nephrectomy [24], it is not clear whether RFA might have influenced the chance of developing this complication, for example by compromising spontaneous healing.

A limitation of this study could be the use of an open approach, which is not the preferable approach for future use. However, the study was designed to accurately study the lesion volume and cell viability within the ablated area, hence the importance of removing the tumor directly following the RFA procedure. Most studies that have used bipolar and multipolar RFA in the treatment of renal tumors have been performed in a percutaneous fashion with a short follow-up, where complete ablation can never be as accurately studied. Now that we have shown that complete ablation of SRMs can be consistently achieved with bipolarmultiprobe RFA, this technique needs to be transferred to a percutaneous approach. Although it cannot be determined whether the renal tumors in our study were all accessible for a percutaneous approach, a few studies have given an indication that this is feasible, and can be performed under CT, MRI, or ultrasound guidance $[18,19,21]$. The precise percutaneous placement of the probes will not always be possible when the tumor is located in an anatomically difficult position. The further development of image-guided techniques will overcome some, but probably not all of these problems. However, further studies are necessary to determine whether it is technically possible to place this number of probes adequately in a percutaneous way.

In conclusion, multipolar RFA is clinically successful for treatment of SRMs and can achieve complete ablation without skip lesions. Using preoperatively calculated energy settings according to tumor size, applied to 1-3 bipolar probes, lesions of tailored sizes can be created. The multipolar use of bipolar probes could make it technically possible to adequately ablate tumors larger than $4 \mathrm{~cm}$. Clinical efficacy and oncological outcomes need to be investigated further in studies using multipolar RFA in a percutaneous fashion.

Conflict of interest Financial and procedural support for the conduction of the study was provided by Olympus Surgical Technologies.

\section{References}

1. Zlotta AR, Wildschutz T, Raviv G et al (1997) Radiofrequency interstitial tumor ablation (RITA) is a possible new modality for treatment of renal cancer: ex vivo and in vivo experience. $\mathrm{J}$ Endourol 11:251

2. Ljungberg B, Bensalah K, Bex A et al (2014) Guidelines on Renal Cell Carcinoma, update April 2014. http://www.uroweb. org/gls/pdf/10\%20Renal\%20Cell\%20Carcinoma_LR.pdf

3. Salas N, Castle SM, Leveillee RJ (2011) Radiofrequency ablation for treatment of renal tumors: technological principles and outcomes. Expert Rev Med Devices 8:695

4. Yoon SK, Choi JC, Cho JH et al (2009) Radiofrequency ablation of renal VX2 tumors with and without renal artery occlusion in a rabbit model: feasibility, therapeutic efficacy, and safety. Cardiovasc Intervent Radiol 32:1241

5. Klingler HC, Marberger M, Mauermann J et al (2007) 'Skipping' is still a problem with radiofrequency ablation of small renal tumours. BJU Int 99:998

6. Michaels MJ, Rhee HK, Mourtzinos AP et al (2002) Incomplete renal tumor destruction using radio frequency interstitial ablation. J Urol 168:2406

7. Gervais DA, Arellano RS, Mueller PR (2005) Percutaneous radiofrequency ablation of renal cell carcinoma. Eur Radiol 15:960

8. Chang I, Mikityansky I, Wray-Cahen D et al (2004) Effects of perfusion on radiofrequency ablation in swine kidneys. Radiology 231:500

9. Goldberg SN, Hahn PF, Tanabe KK et al (1998) Percutaneous radiofrequency tissue ablation: does perfusion-mediated tissue cooling limit coagulation necrosis? J Vasc Interv Radiol 9:101

10. Pereira PL, Trubenbach J, Schenk M et al (2004) Radiofrequency ablation: in vivo comparison of four commercially available devices in pig livers. Radiology 232:482

11. Hacker A, Vallo S, Weiss C et al (2006) Technical characterization of a new bipolar and multipolar radiofrequency device for minimally invasive treatment of renal tumours. BJU Int 97:822

12. Neuhaus J, Blachut L, Rabenalt R et al (2011) Efficiency analysis of bipolar and multipolar radiofrequency ablation in an in vivo porcine kidney model using three-dimensional reconstruction of histologic section series. J Endourol 25:859 
13. Haemmerich D, Wright AW, Mahvi DM et al (2003) Hepatic bipolar radiofrequency ablation creates coagulation zones close to blood vessels: a finite element study. Med Biol Eng Comput 41:317

14. Nakada SY, Jerde TJ, Warner TF et al (2003) Bipolar radiofrequency ablation of the kidney: comparison with monopolar radiofrequency ablation. J Endourol 17:927

15. Osaki Y, Ikeda K, Izumi N et al (2012) Clinical effectiveness of bipolar radiofrequency ablation for small liver cancers. J Gastroenterol 48:874

16. Frericks BB, Ritz JP, Roggan A et al (2005) Multipolar radiofrequency ablation of hepatic tumors: initial experience. Radiology 237:1056

17. Ritz JP, Lehmann KS, Isbert $C$ et al (2006) In-vivo evaluation of a novel bipolar radiofrequency device for interstitial thermotherapy of liver tumors during normal and interrupted hepatic perfusion. J Surg Res 133:176

18. Sommer CM, Lemm G, Hohenstein E et al (2012) CT-guided bipolar and multipolar radiofrequency ablation (RF Ablation) of renal cell carcinoma: specific technical aspects and clinical results. Cardiovasc Intervent Radiol 36:731

19. Boss A, Rempp H, Martirosian P et al (2008) Wide-bore 1.5 Tesla MR imagers for guidance and monitoring of radiofrequency ablation of renal cell carcinoma: initial experience on feasibility. Eur Radiol 18:1449

20. Okhunov Z, Roy O, Duty B et al (2012) Clinical evaluation of a novel bipolar radiofrequency ablation system for renal masses. BJU Int 110:688
21. Salagierski M, Salagierska-Barwinska A, Sosnowski M (2006) Percutaneous ultrasound-guided radiofrequency ablation for kidney tumors in patients with surgical risk. Int J Urol 13:1375

22. Tacke J, Mahnken A, Roggan A et al (2004) Multipolar radiofrequency ablation: first clinical results. Rofo 176:324

23. McCulloch P, Altman D, Campbell W et al (2009) No surgical innovation without evaluation;: the IDEAL recommendations. Lancet 374:1105

24. Ficarra V, Novara G, Secco S et al (2009) Preoperative aspects and dimensions used for an anatomical (PADUA) classification of renal tumours in patients who are candidates for nephron-sparing surgery. Eur Urol 56:786

25. Albrecht D, Germer CT, Isbert C et al (1998) Interstitial laser coagulation: evaluation of the effect of normal liver blood perfusion and the application mode on lesion size. Lasers Surg Med 23:40

26. Veenendaal L, Borel Rinkes I, van Hillegersberg R (2006) Multipolar radiofrequency ablation of large hepatic metastases of endocrine tumours. Eur J Gastroenterol Hepatol 18:89

27. Chang X, Ji C, Zhao X et al (2014) The application of R.e.N.a.L. Nephrometry scoring system in predicting the complications after laparoscopic renal radiofrequency ablation. J Endourol 28:424

28. Seideman C, Gahan J, Weaver M et al (2013) Renal tumour nephrometry score does not correlate with the risk of radiofrequency ablation complications. BJU Int 112:1121 